\title{
ON ANALYTIC NORMAL OPERATORS
}

\section{S. L. JAMISON}

A bounded linear transformation of an abstract Hilbert space $\mathfrak{T}$ into itself will be called an operator on $\mathcal{H C}$. A one-parameter family $T(\epsilon)$ of operators on $\mathfrak{H C}$ will be called regular on the interval $|\epsilon|<\rho$ if it can be expressed as a convergent power series in a real parameter $\epsilon: T(\epsilon)=T_{0}+\epsilon T_{1}+\epsilon^{2} T_{2}+\cdots$, the $T_{k}$ being operators on $\mathcal{F}$. The weak, strong, and uniform convergence of this series are equivalent $[3] .^{1}$

If $S_{n}(\epsilon)$ is the partial sum $T_{0}+\epsilon T_{1}+\cdots+\epsilon^{n} T_{n}, \epsilon$ being real, $S_{n}^{*}(\epsilon)=T_{0}^{*}+\epsilon T_{1}^{*}+\cdots+\epsilon^{n} T_{n}^{*}$. Since a bounded operator has the same norm as its adjoint, $\left\|T^{*}(\boldsymbol{\epsilon})-S_{n}^{*}(\epsilon)\right\|=\left\|T(\epsilon)-S_{n}(\boldsymbol{\epsilon})\right\| \rightarrow 0$ as $n \rightarrow \infty$. Therefore

$$
T^{*}(\epsilon)=T_{0}^{*}+\epsilon T_{1}^{*}+\epsilon^{2} T_{2}^{*}+\cdots .
$$

Thus, if $T(\epsilon)$ is regular and self-adjoint, the self-adjointness of the coefficients $T_{0}, T_{1}, T_{2}, \cdots$ is demonstrated by equating the coefficients of like powers of $\epsilon$ in the series for $T(\epsilon)$ and $T^{*}(\epsilon)$. It is obvious that the self-adjointness of the coefficients implies the self-adjointness of $T(\epsilon)$.

These results do not have a direct analogue for a regular normal operator $N(\boldsymbol{\epsilon})=N_{0}+\epsilon N_{1}+\epsilon^{2} N_{2}+\cdots$ (an operator $N$ is normal if $\left.N N^{*}=N^{*} N\right)$. That is, the normality of the sum does not guarantee the normality of the coefficients nor does the normality of the coefficients imply that the sum is normal. Nevertheless, there is one case -namely when $N(z)=N_{0}+z N_{1}+z^{2} N_{2}+\cdots$ is normal for all (real and complex) $z$ in a neighborhood of $z=0$-where the power series sum being normal guarantees the same for the coefficients. This and more is contained in the corollary to the following theorem.

TheOREM 1. If $A(z)=A_{0}+z A_{1}+z^{2} A_{2}+\cdots$ and $B(z)=B_{0}+z B_{1}$ $+z^{2} B_{2}+\cdots$ converge for $|z|<\rho$, then $A(z) B^{*}(z)=B^{*}(z) A(z)$ for $|z|<\rho$ if and only if $A_{n} B_{m}^{*}=B_{m}^{*} A_{n}$ for every pair of non-negative integers $m$ and $n$.

Proof. By the same reasoning used to obtain (1), $B^{*}(z)=B_{0}^{*}$ $+\bar{z} B_{1}^{*}+\bar{z}^{2} B_{2}^{*}+\cdots$. It is clear that $A(z)$ will commute with $B^{*}(z)$ if each coefficient of $A(z)$ commutes with every coefficient of $B^{*}(z)$, i.e. if $A_{n} B_{m}^{*}=B_{m}^{*} A_{n}$.

Received by the editors July 6, 1953.

${ }^{1}$ Numbers in brackets refer to the bibliography at the end of the paper. 
Suppose now that $A(z)$ commutes with $B^{*}(z)$ for $|z|<\rho$ and let $z=r e^{\theta i}$. Then $\bar{z}=r e^{-\theta i}$. The coefficient of $r^{n+m}$ in $A(z) B^{*}(z)$ is

$$
\sum_{k=0}^{n+m} e^{(n+m-2 k) \theta i} A_{n+m-k} B_{k}^{*}
$$

and in $B^{*}(z) A(z)$ is

$$
\sum_{k=0}^{n+m} e^{(n+m-2 k) \theta i} B_{k}^{*} A_{n+m-k} .
$$

These coefficients must be equal for all values of $\theta$. Writing this down as an identity and multiplying both sides by $e^{(m-n) \theta i}$, the terms involving $A_{n} B_{m}^{*}$ and $B_{m}^{*} A_{n}$ have coefficient 1 and all other terms have coefficients of the form $e^{2 k \theta i}$ where $k$ is a nonzero integer. Thus, on integrating this identity from $\theta=0$ to $\theta=\pi$, the equation $\pi A_{n} B_{m}^{*}$ $=\pi B_{m}^{*} A_{n}$ arises. So $A_{n} B_{m}^{*}=B_{m}^{*} A_{n}$ and the theorem is proved.

CoRollary. If $N(z)=N_{0}+z N_{1}+z^{2} N_{2}+\cdots$ converges for $|z|<\rho$, then $N(z)$ is normal for $|z|<\rho$ if and only if the coefficients $N_{0}, N_{1}$, $N_{2}, \cdots$ form an abelian system of normal operators.

Proof. From the preceding theorem it is seen, on setting $A(z)$ $=B(z)=N(z)$, that $N(z)$ is normal for $|z|<\rho$ if and only if $N_{n} N_{m}^{*}$ $=N_{m}^{*} N_{n}$ for each pair of non-negative integers $m$ and $n$. In particular, $N_{n}$ is normal for each $n$. Moreover, a theorem of B. Fuglede asserts that if a bounded operator commutes with a normal operator, then the adjoint of that bounded operator will also commute with it [1]. (A somewhat simpler proof of this important result has been given by P. Halmos [2].) Thus $N_{n} N_{m}=N_{m} N_{n}$. Hence the coefficients commute with each other and with the adjoints of each other. This is exactly what is meant by an abelian system.

THEOREM 2. If $N(z)=N_{0}+z N_{1}+z^{2} N_{2}+\cdots$ converges and is normal for $|z|<\rho$, then there is a self-adjoint operator $A$ and a function $F(z, \lambda)$ such that

(i) $F(z, \lambda)$ is an analytic function of $z$ for each fixed $\lambda$;

(ii) $F(z, \lambda)$ is A-integrable with respect to $\lambda$ for each $z,|z|<\rho$; and (iii) $N(z)=F(z, A)$.

Proof. By the preceding corollary, the coefficients $N_{0}, N_{1}, N_{2}, \cdots$ form an abelian system of normal operators. Now to each countable abelian system $\left\{N_{k}\right\}$ of normal operators there is a self-adjoint operator $A$ such that each operator $N_{k}$ of the system is a function of $A$, i.e. 


$$
N_{k}=f_{k}(A)=\int_{-\infty}^{\infty} f_{k}(\lambda) d E_{\lambda}
$$

where $\int_{-\infty}^{\infty} \lambda d E_{\lambda}$ is the spectral representation of $A[4$, p. 67]. Take $r<\rho$ and choose $M$ so that $\left\|N_{k}\right\| \leqq M r^{-k}$. Then $\left\|f_{k}(A)\right\|=\left\|N_{k}\right\|$ $\leqq M r^{-k}$. Therefore $\left|f_{k}(\lambda)\right| \leqq M r^{-k}$ for $\lambda$ in the spectrum of $A[5, \mathrm{p}$. 230]. Since $f_{k}(A)$ is independent of the behavior of $f_{k}(\lambda)$ for $\lambda$ in the resolvent set of $A$, it can be assumed that $\left|f_{k}(\lambda)\right| \leqq M r^{-k}$ for all real $\lambda$. Thus $\sum_{k=0}^{\infty} z^{k} f_{k}(\lambda)$ converges absolutely for $|z|<r$. Furthermore, this convergence is uniform with respect to $\lambda$, and hence termwise integration is justified:

$$
\begin{aligned}
\int_{-\infty}^{\infty} \sum_{k=0}^{\infty} z^{k} f_{k}(\lambda) d E_{\lambda} & =\sum_{k=0}^{\infty} z^{k} \int_{-\infty}^{\infty} f_{k}(\lambda) d E_{\lambda}=\sum_{k=0}^{\infty} z^{k} f_{k}(A) \\
& =\sum_{k=0}^{\infty} z^{k} N_{k}=N(z) .
\end{aligned}
$$

Since $r$ can be taken arbitrarily close to $\rho$, it follows on setting $F(z, \lambda)$ $=\sum_{k=0}^{\infty} z^{k} f_{k}(\lambda)$ that (i), (ii), and (iii) of the theorem are satisfied.

\section{REFERENCES}

1. B. Fuglede, A commutativity theorem for normal operators, Proc. Nat. Acad. Sci. U.S.A. vol. 36 (1950) pp. 35-40.

2. P. R. Halmos, Commutativity and spectral properties of normal operators, Acta Szeged. vol. 12 (1950) pp. 153-156.

3. S. L. Jamison, Perturbation of normal operators, Proc. Amer. Math. Soc. vol. 5 (1954) pp. 103-110.

4. B. v. Sz. Nagy, Spektraldarstellung linearer Transformationen des Hilbertschen Raumes, Berlin, 1942.

5. M. H. Stone, Linear transformations in Hilbert space and their applications to analysis, Amer. Math. Soc. Colloquium Publications, vol. 15, 1932.

Florida State University 